\title{
CÔNG TÁC CHİNH LÝ BẢN ĐỒ SỐ ĐỊA HÌNH, ĐỊA CHÍNH
}

\author{
ĐINH CÔNG HÒA \\ Trường Đại học Mỏ - Địa chất
}

\section{Tóm tắt:}

Trên co sở các thiết bị mới, hiện đại ngày càng được ưng dụng rộng rãi trong sản xuất trắc địa cùng với sự phát triển của công nghệ tin hoc, tác giả đưa ra giải pháp công nghệ và xây dụng môđun chưong trình úng dụng nhằm tụ động hóa công tác biên tập chỉnh lý bản đồ số trên cơ sở các số liệu thu thập tù thiết bị: Toàn đạc điện tử; Đo động thời gian thưc RTK (real time kinematic); Trạm tham chiếu hoạt động liên tục CORS (Continously Operating Reference Station). Bằng ngôn ngũ lập trình $C++, V B 6.0$ và lý thuyết sai số, thuật toán biến đổi tọa độ trên mặt phẳng, tác giả xây dụng mô-đun chưong trình tụ động công tác chỉnh lý bản đồ đáp ứng yêu cầu về độ chính xác đặt ra với hiệu quả kinh tế cao. Kết quả nghiên cứu là mô-đun chuơng trình được kiểm chứng tính chinh xác thông qua các số liệu thực tế.

\section{1. Đặt vấn đề}

Bản đồ địa hình phục vụ cho công tác khảo sát, thiết kế, xây dựng và quy hoạch... Bản đồ địa chính là tài liệu cơ bản phục vụ cho ngành quản lý đất đai trong các công tác như đăng ký thống kê đất đai, quy hoạch phân bổ sử dụng đất, thanh tra - kiểm tra đất đai, cấp giấy chứng nhận quyền sử dụng đất... Vì vậy bản đồ số cần phải được quản lý chặt chẽ, có hệ thống và liên tục được cập nhật theo yêu cầu của thực tế.

Trong thực tế do nhiều nguyên nhân khác nhau mà ranh giới, hình thể thửa đất có sự thay đổi vì thế phải theo dõi và chỉnh lý kịp thời, thường xuyên. Chỉnh lý bản đồ là đảm bảo cho hình thể có trên bản đồ luôn phù hợp với hình thể có ngoài thực địa, Theo phương pháp truyền thống, đối với cấp xã phường khi chỉnh lý bản đồ địa chính thường dùng các phương pháp giao hội cạnh; phương pháp đường thẳng hàng, chọn các điểm làm gốc là những điểm rõ nét có trên bản đồ địa chính và tương ứng của chúng ở ngoài thực địa .

Hiện nay, với những tiến bộ khoa học đang được ứng dụng rộng rãi trong sản xuất trắc địa, việc hiện chỉnh bản đồ số đã ứng dụng máy toàn đạc điện tử, phương pháp đo RTK hoặc phương pháp CORS để đo vẽ lại một khu vực khi có sự thay đổi lớn. Trong các trường hợp này chúng ta cần phải có các điểm khống chế đo vẽ gần khu vực đo đạc chỉnh lý, điều này dẫn đến nhiều khó khăn về mặt kỹ thuật và kinh tế.

Để khắc phục những khó khăn trong việc xây dựng hoặc khôi phục các điểm khống chế đo vẽ khu vực đo chỉnh lý, chúng ta áp dụng bài toán đo chỉnh lý biến động trong hệ thống tọa độ giả định. Trong hệ thống tọa độ giả định, hình thể của các đối tượng biến động đã được xác định một cách chính xác theo yêu cầu tương ứng với tỷ lệ bản đồ. Để kết nối các đối tượng đo chỉnh lý với bản đồ trong cùng một hệ thống tọa độ, ngoài việc đo đạc các đối tượng biến động, chúng ta đo nối đến một số điểm rõ nét có trên bản đồ hoặc các điểm khống chế còn tồn tại gần khu vực chỉnh lý. Trên cơ sở tọa độ các điểm rõ nét trên bản đồ hoặc điểm khống chế tọa độ và tọa độ có được trong hệ tọa độ giả định (điểm song trùng), thông qua bài toán biến đổi tọa độ trên mặt phẳng gồm: Phép biến đổi Affine, Helmert, Biến đổi đa thức, xác định các hệ số biên đổi theo nguyên lý số bình phương nhỏ nhất, tính chuyển tọa độ các điểm đo chỉnh lý về hệ tọa độ bản đồ.

Quá trình tính toán chuyển đổi được thực hiện thông qua một mô-đun chương trình trong phần mềm GeoSoft mà tác giả đã xây dựng phục 
vụ cho thực tế sản xuất và đào tạo trong nhiều năm qua. Tính toán thực nghiệm cho thấy phương án đo chỉnh lý và mô-đun chỉnh lý bản đồ hoàn toàn phù hợp với thực tế sản xuất, đạt yêu cầu về độ chính xác và hiệu quả kinh tế.

\section{Bài toán biến đổi tọa độ trên mặt phẳng}

Biến đổi tọa độ phẳng từ hệ $(\mathrm{xoy})_{1}$ sang hệ (xoy) $)_{2}$ được thực hiện theo công thức [2]. Hoàng Ngọc Hà. 2001. Tính toán trắc địa và cơ sở dư liệu) bao gồm:

a-Phép biến đổi Affine.

$$
\begin{aligned}
& \mathrm{x}_{2}=\mathrm{ax}_{1}+\mathrm{by}_{1}+\mathrm{c} \\
& \mathrm{y}_{2}=\mathrm{dx}_{1}+\mathrm{ey}_{1}+\mathrm{g}
\end{aligned}
$$

\section{b-Phép biến đổi Helmert.}

$\mathrm{x}_{2}=\mathrm{x}_{0}+\mathrm{m} \cdot \cos \alpha \cdot \mathrm{x}_{1}-\mathrm{m} \cdot \sin \alpha \cdot \mathrm{y}_{1}$

$$
\mathrm{y}_{2}=\mathrm{y}_{0}+\mathrm{m} \cdot \sin \alpha \cdot \mathrm{x}_{1}-\mathrm{m} \cdot \cos \alpha \cdot \mathrm{y}_{1}
$$

$\mathrm{m}=1+\Delta \mathrm{m}$,

$\Delta \mathrm{m}$ : sự thay đổi tỷ lệ xích

$\alpha$ : góc xoay giữa hai trục tọa độ của hai hệ

c-Phép biến đổi đa thức bậc 2.

$\mathrm{x}_{2}=\mathrm{a}_{1}+\mathrm{b}_{1} \mathrm{x}_{1}+\mathrm{c}_{1} \mathrm{y}_{1}+\mathrm{d}_{1} \mathrm{x}_{1}^{2}+\mathrm{e}_{1} \mathrm{y}_{1}^{2}+\mathrm{g}_{1} \mathrm{x}_{1} \mathrm{y}_{1}$

$\mathrm{y}_{2}=\mathrm{a}_{2}+\mathrm{b}_{2} \mathrm{x}_{1}+\mathrm{c}_{2} \mathrm{y}_{1}+\mathrm{d}_{2} \mathrm{x}_{1}^{2}+\mathrm{e}_{2} \mathrm{y}_{1}^{2}+\mathrm{g}_{2} \mathrm{x}_{1} \mathrm{y}_{1}(3)$

$d$ - Xác định hệ số biến đổi dựa vào tọa độ các điểm song trùng giũa hai hệ tọa độ.

Đối với phép biến đổi Affine chúng ta cần xác định 6 hệ số: a,b,c,d,e,g; Phép biến đổi Helmert cần xác định 4 hệ số: $\mathrm{x}_{0}, \mathrm{y}_{0}, \mathrm{a}, \mathrm{b}$; Phép biến đổi đa thức bậc 2 cần xác định 12 hệ số: $\mathrm{a}_{1}$, $b_{1}, c_{1}, d_{1}, e_{1}, g_{1}, a_{2}, b_{2}, c_{2}, d_{2}, e_{2}, g_{2}$. Như vậy tùy thuộc vào số lượng điểm song trùng chúng ta lựa chọn bài toán biến đôi tọa độ. Phép biến đổi Helmert cần tối thiểu 2 điểm song trùng, phép biến đổi Affine cần tối thiểu 3 điểm và phép biến đổi đa thức bậc 2 cần tối thiểu 6 điểm. Khi số điểm song trùng lớn hơn số điểm tối thiểu, chúng ta xác đinh các hệ số theo nguyên lý số bình phương nhỏ nhất [1]. Hoàng Ngọc Hà, Trương
Quang Hiếu. 1999. Co' sở toán học xủ lý số liệu trắc địa).

Để xác định các hệ số biến đổi chúng ta áp dụng bài toán bình sai gián tiếp, ẩn số là các hệ số biến đổi. Mỗi cặp điểm song trùng, chúng ta viết được 2 phương trình số hiệu chỉnh tọa độ $\mathrm{x}$ và $\mathrm{y}$, cụ thể như sau:

Phép biến đổi Affine:

$$
\begin{aligned}
& v_{x}=x_{1} \cdot a+y_{1} \cdot b+c+1_{x} \\
& v_{y}=x_{1} \cdot d+y_{1} \cdot e+g+1_{y}
\end{aligned}
$$

Phép biến đổi Helmert:

$$
\begin{aligned}
& \mathrm{v}_{\mathrm{x}}=\mathrm{x}_{1} \cdot \mathrm{a}-\mathrm{y}_{1} \cdot \mathrm{b}+\mathrm{x}_{0}+\mathrm{1}_{\mathrm{x}} \\
& \mathrm{v}_{\mathrm{y}}=\mathrm{y}_{1} \cdot \mathrm{a}+\mathrm{x}_{1} \cdot \mathrm{b}+\mathrm{y}_{0}+\mathrm{1}_{\mathrm{y}}
\end{aligned}
$$

Phép biến đổi đa thức bậc 2 :

$$
\begin{aligned}
\mathrm{v}_{\mathrm{x}}= & \mathrm{a}_{1}+\mathrm{x}_{1} \cdot \mathrm{b}_{1}+\mathrm{y}_{1} \cdot \mathrm{c}_{1}+\mathrm{x}_{1}{ }^{2} \cdot \mathrm{d}_{1}+ \\
& +\mathrm{y}_{1}{ }^{2} \cdot \mathrm{e}_{1}+\mathrm{x}_{1} \mathrm{y}_{1} \cdot \mathrm{g}_{1}+\mathrm{l}_{\mathrm{x}} \\
\mathrm{v}_{\mathrm{y}}= & \mathrm{a}_{2}+\mathrm{x}_{1} \cdot \mathrm{b}_{2}+\mathrm{y}_{1} \cdot \mathrm{c}_{2}+\mathrm{x}_{1}{ }^{2} \cdot \mathrm{d}_{2}+ \\
& +\mathrm{y}_{1}{ }^{2} \cdot \mathrm{e}_{2}+\mathrm{x}_{1} \mathrm{y}_{1} \cdot \mathrm{g}_{2}+\mathrm{l}_{\mathrm{y}}
\end{aligned}
$$

trong đó: $1_{\mathrm{x}}=-\mathrm{x}_{2} ; 1_{\mathrm{y}}=-\mathrm{y}_{2}$

Như vậy chúng ta có hệ phương trình số hiệu chỉnh (số phương trình bằng 2 lần số điểm song trùng) viết dưới dạng ma trận:

$$
\mathrm{V}=\mathrm{A} \Delta \mathrm{x}+\mathrm{L}
$$

Trên cơ sở hệ phương trình số hiệu chỉnh, theo nguyên lý số bình phương nhỏ nhất $[\mathrm{pvv}]=$ min, chúng ta lập được phương trình chuẩn dạng:

$\mathrm{R} \Delta \mathrm{x}+\mathrm{B}=0$; trong đó $\mathrm{R}=\mathrm{A}^{\mathrm{T}} \mathrm{A} ; \mathrm{B}=\mathrm{A}^{\mathrm{T}} \mathrm{L}$

Giải hệ phương trình chuẩn được các ẩn số là các hệ số biến đổi:

$$
\Delta \mathrm{x}=-\mathrm{R}^{-1} \mathrm{~B}
$$

Sau khi xác định được các hệ số biến đổi, chúng ta tính được tọa độ các điểm đo chỉnh lý trong hệ tọa độ giả định về hệ tọa độ bản đồ theo các công thức (1), (2), (3) tùy theo số lượng điểm song trùng. 


\section{Mô đun chương trình chỉnh lý bản đồ}

Để quá trình chỉnh lý bản đồ được thực hiện một cách nhanh chóng và chính xác, chúng tôi đã xây dựng được mô-đun chương chỉnh lý bản đồ theo sơ đồ khối nêu trong hình 1 [3]. Đinh Công Hòa. 2011. Lập trình bài toán trắc địa co $\left.s o^{\circ}\right)$.

Số liệu đầu vào là bản đồ số đo chỉnh lý trong hệ tọa độ giả định bằng máy toàn đạc điện tử, phương pháp đo RTK hoặc phương pháp CORS và tọa độ các điểm điểm song trùng. Đầu ra của chương trình là các hệ số biến đổi và bản đồ đo chỉnh lý trong hệ tọa độ bản đồ thành lập.

Để có được bản đồ số sau chỉnh lý. Từ bản đồ gốc chúng ta xóa bỏ hoặc lưu lại các đối tượng lạc hậu hoặc không còn tồn tại ngoài thực địa thông qua lớp thông tin lưu trữ. Bằng kỹ thuật dữ liệu đồ họa chuẩn, chúng ta dễ dàng ghép nối bản đồ đo chỉnh lý đã hiệu chỉnh về hệ tọa độ bản đồ gốc với bản đồ gốc để có được bản đồ mới sau chỉnh lý thông qua các phần mềm đồ họa AutoCad, MicroStation ...

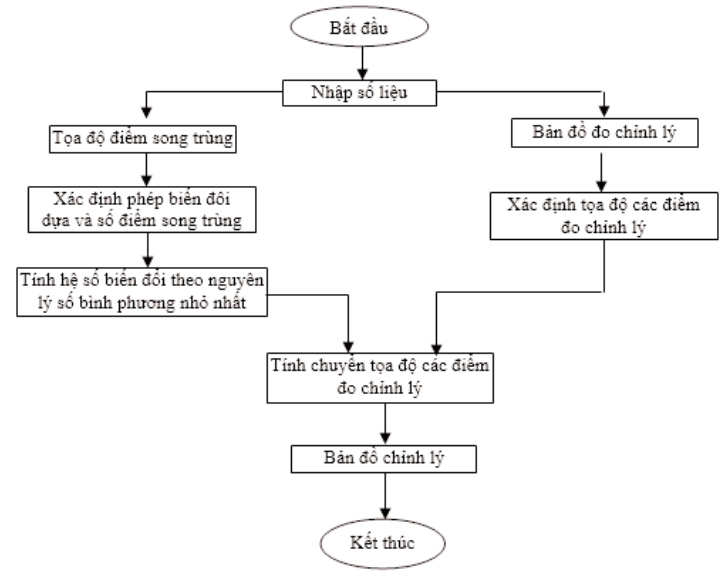

Hình 1: So đồ khối mô-đun chương trình chỉnh lý bản đồ

Mô-đun chương trình được xây dựng bằng ngôn ngữ VB 6.0, các chỉ dẫn thực hiện bằng tiếng Việt, thuận lợi cho người sử dụng (hình 2).

\section{Kết quả thực nghiệm}

Để kiểm định tính ưu việt, độ chính xác của bài toán và mô-đun chương trình đã nêu, bằng số liệu thực tế của mảnh bản đồ tỷ lệ $1 / 200$, tiến hành đo chỉnh lý một khu vực trong hệ tọa độ giả định bằng máy toàn đạc điện tử. Trong khu vực đo chỉnh lý, lựa chọn 4 điểm ổn định và rõ nét (ST1, ST2, ST3, ST4), các điểm này có tọa độ trong hệ tọa độ giả định và trên bản đồ đã thành lập. (Hinh 3)

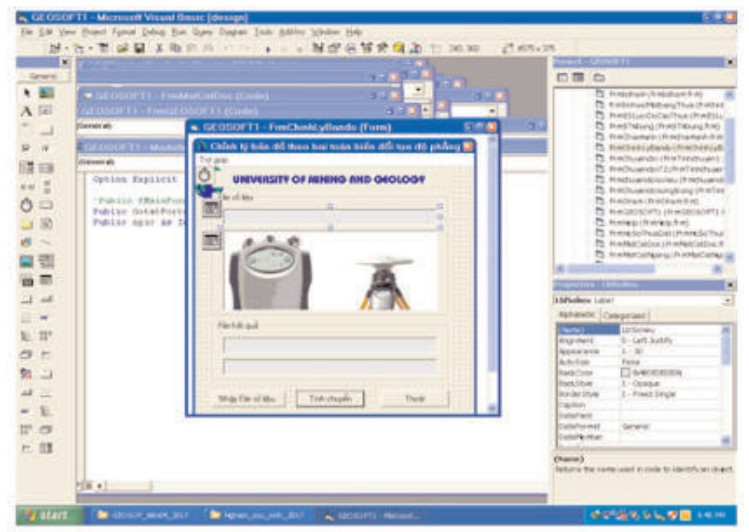

Hình 2: Mô đun chuoong trình chinh lý bản đồ

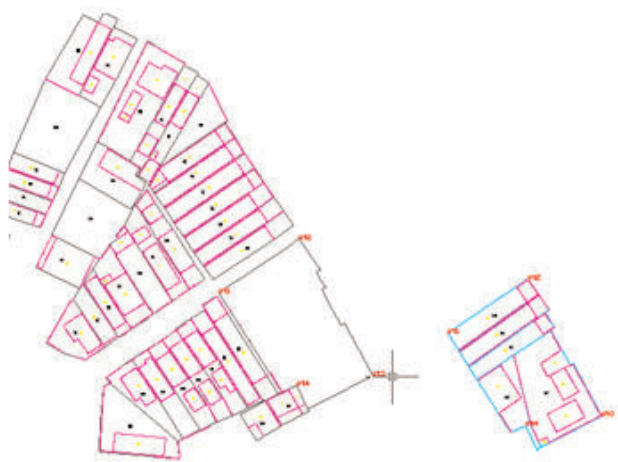

\section{Hình 3: Số liệu thực nghiệm: Bản đồ thành lập và bản vẽ đo chinh lý}

Bằng thuật toán biến đổi tọa độ phẳng thông qua mô-đun chương trình, xác định các hệ số biến đổi, thông qua hệ số biến đổi, hiệu chỉnh lại bản vẽ đo chỉnh lý về bản đồ đã thành lập. Số liệu tính toán nêu trong bảng 1 , bản đồ sau chỉnh lý nêu trong hình 4.

\section{Kết luận}

Bằng thuật toán, mô-đun chương trình và kết quả thực nghiệm đã chứng tỏ tính đúng đắn của vấn đề mà tác giả đã đưa ra. Mô-đun chương trình chỉnh lý bản đồ hoàn toàn đủ điều kiện để 
áp dụng trong sản xuất nâng cao hiệu quả kinh tế, kỹ thuật. Độ chính xác các điểm đo chỉnh lý khi chuyển về bản đồ thành lập chỉ còn phụ thuộc vào độ chính xác các điểm đo chi tiết trong hệ tọa độ giả định, độ chính xác đo tọa độ các điểm song trùng và số lượng điểm song trùng. $\bigcirc$

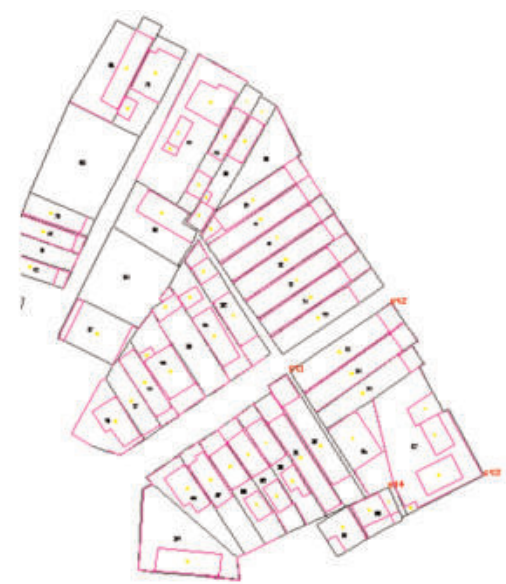

Hình 4: Bản đồ thành lập sau đo vẽ chỉnh lý

\section{Tài liệu tham khảo}

[1]. Hoàng Ngọc Hà, Trương Quang Hiếu. 1999. Co sơ toán họ xư lý số liệu trắc địa. Nhà xuất bản Giao thông vận tải. Hà Nội

[2]. Hoàng Ngọc Hà. 2001. Tính toán trắc địa và $c o$ sở dĩ liệ. Nhà xuất bản Giao thông vận tải. Hà Nội

[3]. Đinh Công Hòa. 2011. Lập trình bài toán trắc địa cơ sở. Nhà xuất bản Giao thông vận tải. Hà Nội.O

\section{Summary}

\section{Editing topographical map and cadastral map}

\section{Dinh Cong Hoa}

\section{Hanoi University of Mining and Geology}

Recently, many advanced equipments are widely applied in surveying along with the development of the information technology. Therefore, this study proposed a solution and built a programmed module for automatic editing cadastral map based on data collected from a number of advanced equipments, such as: Electronic Total Station; GNSS-real time kinematic (RTK); Continuously Operating Reference Stations (CORS). By combining C ++ language, VB 6.0 language, error theory and coordinate transformation algorithm, the programmed module for automatic cadastral map editing was generated. The experimental results showed that the module is efficiency and ensure editing accuracy. $\bigcirc$

\section{Bảng 1: Kết quả tính hệ số biến đổi tọa độ phẳng}

\begin{tabular}{|c|c|c|c|c|c|}
\hline \multirow{2}{*}{$\begin{array}{c}\text { Điểm } \\
\text { song } \\
\text { trùng }\end{array}$} & \multicolumn{2}{|c|}{ Tọa độ trên bản đồ } & \multicolumn{2}{|c|}{ Tọa độ đo chỉnh lý } & \multirow{2}{*}{ Các hệ số hiến đồi sau tính toán } \\
\hline & $\mathrm{X}$ & $\mathrm{Y}$ & $\mathrm{X}$ & $\mathrm{Y}$ & \\
\hline St1 & 2321017.513 & 501112.125 & 2321026.492 & 501060.707 & $\mathrm{~A}=1.00003355 ; \mathrm{B}=0.00001821$ \\
\hline St2 & 2321029.504 & 501130.110 & 2321038.484 & 501078.691 & $\mathrm{C}=2321019.706000000000$ \\
\hline $\mathrm{St} 3$ & 2320999.004 & 501146.654 & 2321007.983 & 501095.235 & $\mathrm{D}=-0.000004058, E=0.99996854$ \\
\hline St4 & 2320996.886 & 501129.646 & 2321005.865 & 501078.227 & $\mathrm{G}=501078.215000000000$ \\
\hline
\end{tabular}

